\title{
Burkholderia pseudomallei transcriptional adaptation in macrophages
}

\author{
Sylvia Chieng ${ }^{1}$, Laura Carreto ${ }^{2}$ and Sheila Nathan ${ }^{1 *}$
}

\begin{abstract}
Background: Burkholderia pseudomallei is a facultative intracellular pathogen of phagocytic and non-phagocytic cells. How the bacterium interacts with host macrophage cells is still not well understood and is critical to appreciate the strategies used by this bacterium to survive and how intracellular survival leads to disease manifestation.

Results: Here we report the expression profile of intracellular B. pseudomallei following infection of human macrophage-like U937 cells. During intracellular growth over the $6 \mathrm{~h}$ infection period, approximately $22 \%$ of the $B$. pseudomallei genome showed significant transcriptional adaptation. B. pseudomallei adapted rapidly to the intracellular environment by down-regulating numerous genes involved in metabolism, cell envelope, motility, replication, amino acid and ion transport system and regulatory function pathways. Reduced expression in catabolic and housekeeping genes suggested lower energy requirement and growth arrest during macrophage infection, while expression of genes encoding anaerobic metabolism functions were up regulated. However, whilst the type $\mathrm{VI}$ secretion system was up regulated, expression of many known virulence factors was not significantly modulated over the 6 hours of infection.
\end{abstract}

Conclusions: The transcriptome profile described here provides the first comprehensive view of how $B$. pseudomallei survives within host cells and will help identify potential virulence factors and proteins that are important for the survival and growth of B. pseudomallei within human cells.

Keywords: Burkholderia pseudomallei, Macrophage, Transcriptome analysis

\section{Background}

Burkholderia pseudomallei causes melioidosis, a disease with considerable human mortality and morbidity in the tropics [1]. Clinical presentation of melioidosis varies from rapidly fatal septicemia and acute pneumonia, chronic or localized abscess formation to skin and soft tissue infections that progress rapidly to systemic infection [2,3]. Treatment of melioidosis involves long periods of antibiotic administration which are vital to eliminate B. pseudomallei and to prevent relapse $[4,5]$.

Several features of melioidosis suggest that B. pseudomallei is a facultative intracellular bacterium. These include long incubation periods of up to 62 years and the tendency to relapse $[3,6]$. B. pseudomallei has the ability to survive and proliferate within phagocytic and non-

\footnotetext{
* Correspondence: sheila@ukm.my

'School of Biosciences and Biotechnology, Faculty of Science and Technology, Universiti Kebangsaan Malaysia, Bangi 43600, Malaysia Full list of author information is available at the end of the article
}

phagocytic cells $[7,8]$, and free-living amoeba [9] for months or years. The exact mechanism of invasion is still unknown, but it requires the rearrangement of host actin cytoskeleton and the involvement of BopE, an effector protein of the type III secretion system (T3SS-3) [10]. After cellular uptake, B. pseudomallei escapes from endocytic vacuoles and once in the cytoplasm, induces host cell fusion and enters neighbouring cells by forming actin tails and membrane protrusions [11,12]. Vacuole escape and intracellular survival requires a functional T3SS-3, as mutants of T3SS-3 display delayed vacuolar escape, reduced actin formation and reduced capacity to survive intracellularly and spread to neighbouring cells $[13,14]$. It is possible that intracellular survival and intercellular spread may provide B. pseudomallei protection from host defences.

Recently, the type VI secretion cluster tss-5 (T6SS-1) was shown to contribute to intracellular growth of $B$. pseudomallei [15]. The expression of this secretion

\section{() Biomed Central}


system is dependent on the sensor regulators $\mathrm{BprC}$ and VirA-VirG (VirAG). The type VI secretion system is also thought to play a major role during bacterial transition from the phagosome to the cytosol [15]. Moreover, T6SS is also implicated in other important bacterial pathogens as the key virulence factor and is involved in translocation of effector proteins into eukaryotic cells $[16,17]$. Using in vivo expression technology, Shalom et al. [18] found that the B. pseudomallei tss-5 gene cluster was induced inside murine macrophages. Furthermore, Pilatz and colleagues demonstrated that a B. pseudomallei tss-5 transposon mutant displayed reduced ability to form plaques on PtK2 epithelial cell monolayers, indicating the requirement of tss-5 in cellto-cell spread [19]. This mutant was also highly attenuated in mice with reduced bacterial load in the spleen, liver and lung at $48 \mathrm{~h}$ post-infection [19].

Previous studies have examined the detailed cellular responses of $B$. pseudomallei within eukaryotic cells. However, the overall picture of the mechanisms involved in adaptability to the intracellular lifestyle is still unclear. The availability of the complete genome sequence for $B$. pseudomallei [20] enabled us to design a whole genome DNA microarray to identify B. pseudomallei genes regulated during infection of macrophages. These data provide an insight into genes involved in survival and adaptation of the bacteria within macrophage cells as well as a new understanding of the biology of hostpathogen interaction during melioidosis.

\section{Results}

\section{Infection model and bacterial RNA isolation}

Human macrophage-like U937 cells were chosen as the infection model as it has previously been used extensively to study $B$. pseudomallei interaction with host cells $[7,8]$. In this study, bacterial replication within macrophages was observed with a maximum CFU obtained at $6 \mathrm{~h}$ post-infection. The calculated doubling time for intracellular B. pseudomallei is about $6.4 \mathrm{~h}$, indicating a slower growth rate compared to the control bacteria in RPMI (doubling time of $\sim 1.9 \mathrm{~h}$ ). The number of viable bacteria decreased consistently after $6 \mathrm{~h}$ postinfection and a $2-\log$ reduction in viable cells was noted with prolonged incubation of up to $24 \mathrm{~h}$. A similar decline in bacterial numbers following prolonged incubation was previously reported in RAW264.7 cells $[7,21]$. Viability of infected U937 cells decreased over time and was drastically reduced by $6 \mathrm{~h}$ post-infection, suggesting a cytotoxic effect of $B$. pseudomallei on the host cells (Figure 1).

An ultrastructural study of infected U937 cells showed that the nuclei, mitochondria and vacuoles were swollen whilst the nuclear material appeared diluted and the cell membrane totally disrupted (Figure 2A). All these

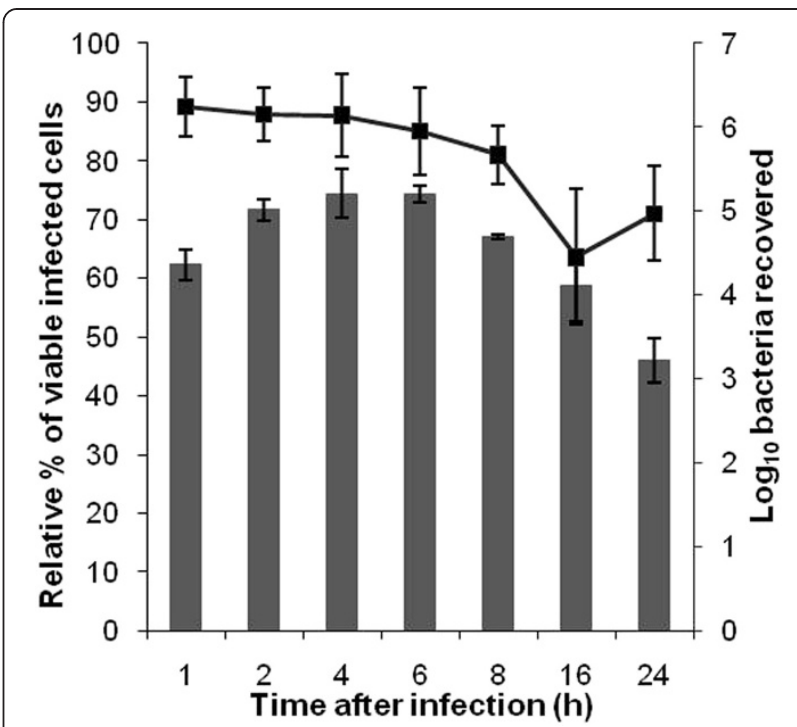

Figure $1 \mathrm{~B}$. pseudomallei intracellular growth and viability of infected U937. Intracellular survival of B. pseudomallei was monitored over a $24 \mathrm{~h}$ period, along with U937 cell viability after B. pseudomallei infection. The relative amount of bacteria recovered $\left(\log _{10}\right.$ scale) was plotted in grey bars while the relative $\%$ of viable infected cells was indicated as a solid line. Vertical lines represent the standard deviation (SD) obtained from three independent experiments conducted in triplicate $(n=9)$.

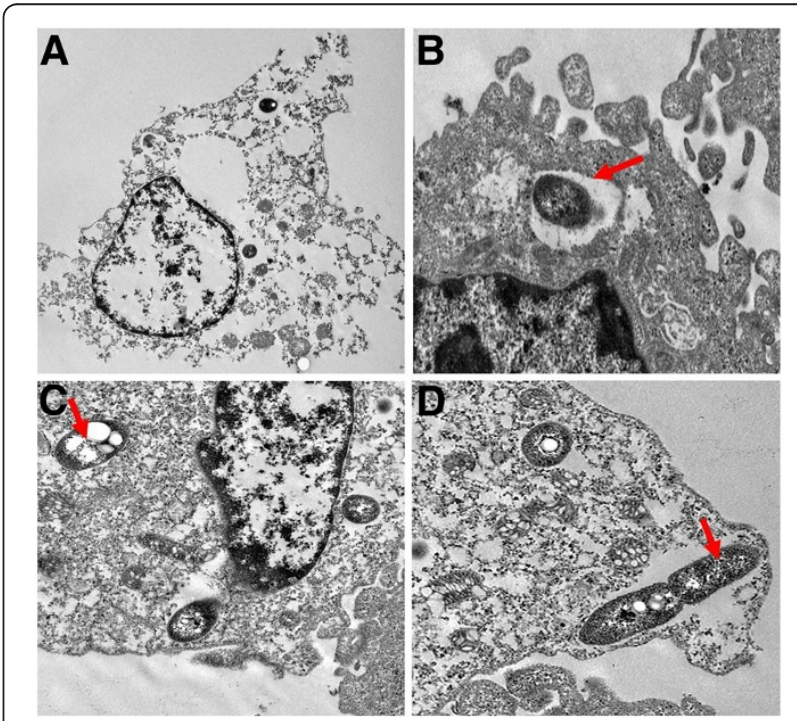

Figure 2 Transmission electron micrograph of intracellular $B$. pseudomallei within U937 cells. (A) B. pseudomallei-infected U937 cells demonstrating oncotic-like morphology and disruption of cell membrane. $\times 7000$ (B) Internalised B. pseudomallei within membranebound phagosome (arrowed) were observed at early stage of intracellular infection. $\times 8000$ (C) Loss of phagosome membrane was clearly visible and free bacteria were seen in the cell cytoplasm (arrowed). $\times 8000$ (D) A dividing bacillus in a phagosome with nearintact membrane (arrowed). Some membrane dissolution can be seen close to the ends of the bacterial cell. $\times 8000$. 
changes indicate that infected U937 cells displayed a phenotype similar to oncotic cells as previously observed in other cells infected with B. pseudomallei [21]. Loss of phagosome membrane in $B$. pseudomallei infected macrophage cells was established as early as $15 \mathrm{~min}$ post-incubation similar to that previously reported [22]. At $2 \mathrm{~h}$ post-infection, loss of phagosome membrane is evident and bacilli were seen in the cytoplasm (Figure 2C). Moreover, replication of intracellular bacteria was seen in the cytoplasm of host cells (Figure 2D).

An infection period of 1 to $6 \mathrm{~h}$ was selected to study changes in the expression profile as more than $85 \%$ of the infected U937 cells were still viable during this period (Figure 1). This would ensure maximum recovery of intracellular B. pseudomallei from viable cells to obtain sufficient intact bacterial RNA. We adopted the differential lysis method with saponin to prevent bacterial cell lysis and easily recover bacterial RNA. This strategy enriches the bacterial mRNA several thousand-fold [23]. Treatment with saponin specifically lyses eukaryotic cells without affecting bacterial viability $[24,25]$ and in our study, the use of $0.1 \%$ to $4 \%$ saponin was not detrimental to B. pseudomallei (data not shown). Differential centrifugation was combined with this method to efficiently remove host cellular debris. Based on the RNA integrity number (RIN) and the $16 \mathrm{~S} / 23 \mathrm{~S}$ rRNA subunit ratio, the electrophoretic profiles of the total RNA extracted from intracellular bacteria showed no evidence of degradation and only minor host RNA contamination was detectable (Figure 3).

\section{The global gene expression profile}

Temporal gene expression profiles obtained from intracellular B. pseudomallei were compared with the transcriptome of control bacteria grown in cell culture medium. Statistical analysis $(p<0.01)$ combined with a 2 -fold variation cut off indicated that 2,797 genes were differentially expressed at $1 \mathrm{~h}, 2,755$ at $2 \mathrm{~h}, 2,776$ at $4 \mathrm{~h}$ and 1,918 genes at $6 \mathrm{~h}$. Hierarchical clustering of gene expression levels revealed similar patterns of gene regulation over the period of infection (Figure 4A). The majority of the genes with altered expression were downregulated throughout the infection period compared to bacteria grown in control medium. Expression levels of these genes continued to decrease up to $4 \mathrm{~h}$ before increasing to levels lower or similar to control bacteria at 6 h. It appears that the adaptation of B. pseudomallei within U937 cells is rapid and most of the changes occurred as early as $1 \mathrm{~h}$ post-infection (Figure 5). With time, the number of significantly deregulated genes gradually decreased, suggesting that B. pseudomallei had become adapted to the intracellular environment. Functional classification of intracellularly modulated bacterial genes at each time point showed that most of

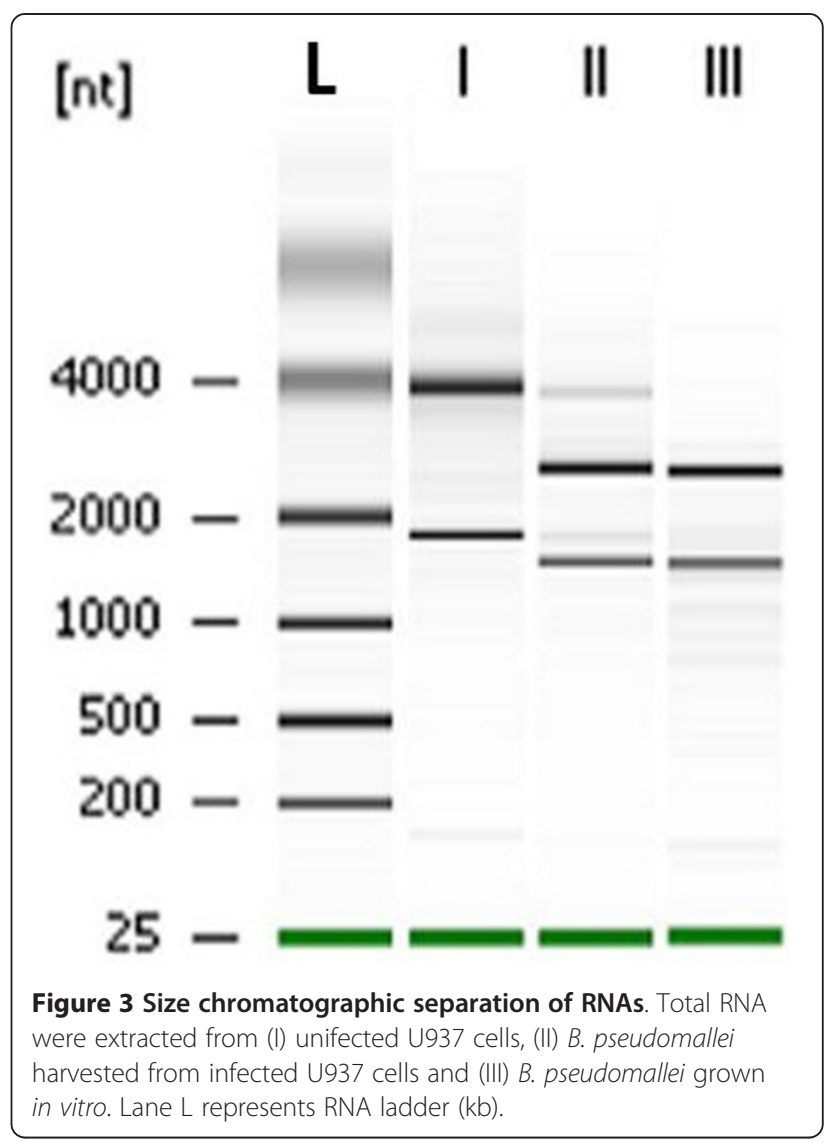

these genes encoded core functions such as metabolism, cell envelope, regulatory functions, transport and binding. Many genes encoding proteins with unknown function or hypothetical proteins were also modulated during infection (Figure 6).

Of the 5,721 probes represented on our microarrays, 1,284 transcripts representing $22.4 \%$ of the B. pseudomallei ORFeome, were differentially expressed during the infection time course. Of these, 25 genes demonstrated increased expression (Table 1) while 1,259 genes were down-regulated (Additional file 1). We performed gene function enrichment analysis on these genes to further categorize them into biological functions. Based on the statistical analysis of KEGG biochemical pathways, the number of genes involved in cellular functions such as biosynthesis of flagella and capsule, energy metabolism and regulatory systems was significantly higher during intracellular infection (Table 2).

As an independent measure of differential gene expression, we examined the relative expression of six upor down-regulated genes selected from different functional categories by real-time qPCR (Table 1 and Additional file 1) on the same samples as those used for microarray analysis. Changes in expression were verified by real-time qPCR with a correlation of $>0.95$ to the microarray data (Figure 7). The strong correlation 


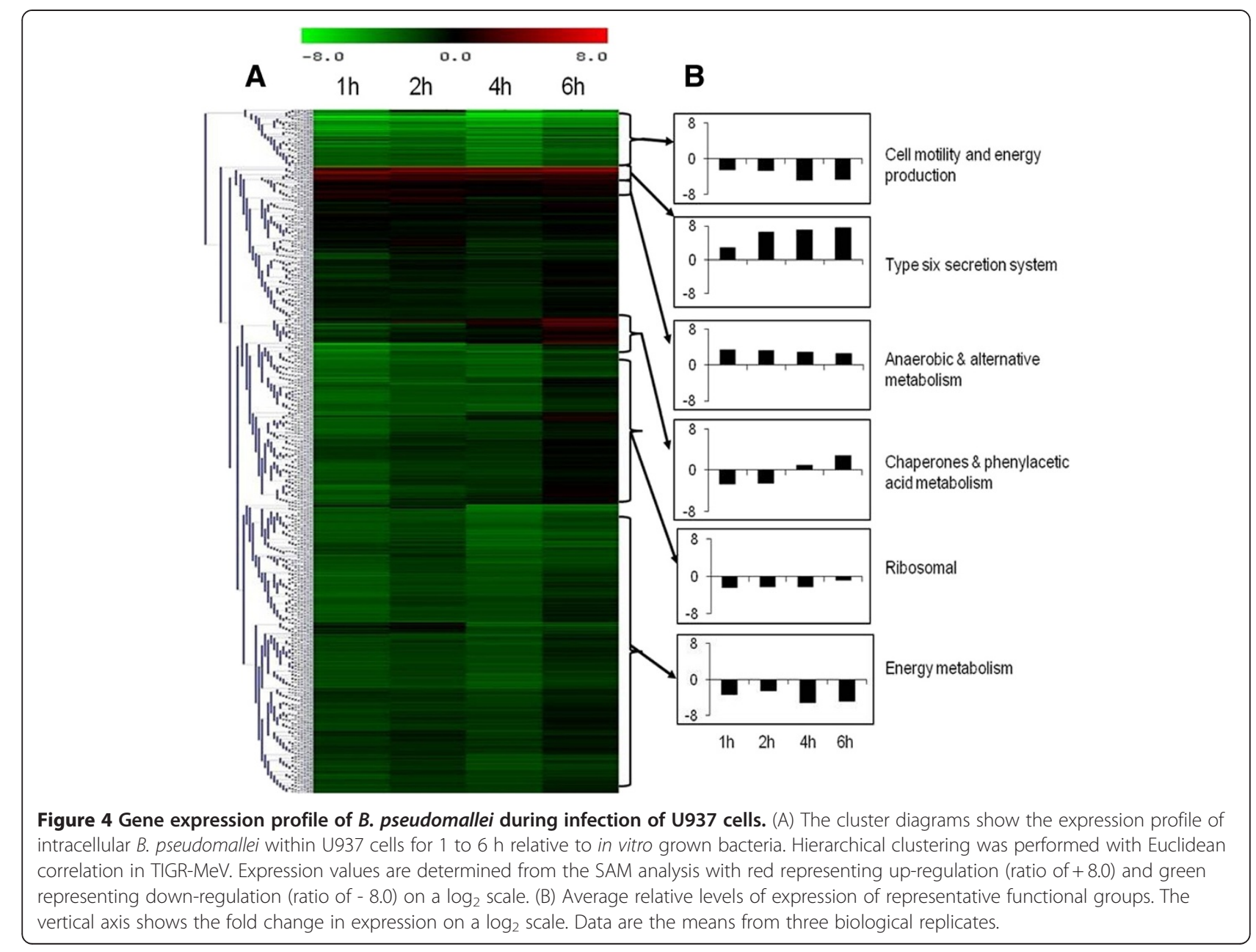

observed verified the efficiency and robustness of the designed microarray for high throughput screening of the B. pseudomallei transcriptome.

Due to the large number of significantly differentiated genes modulated during the infection, only data related

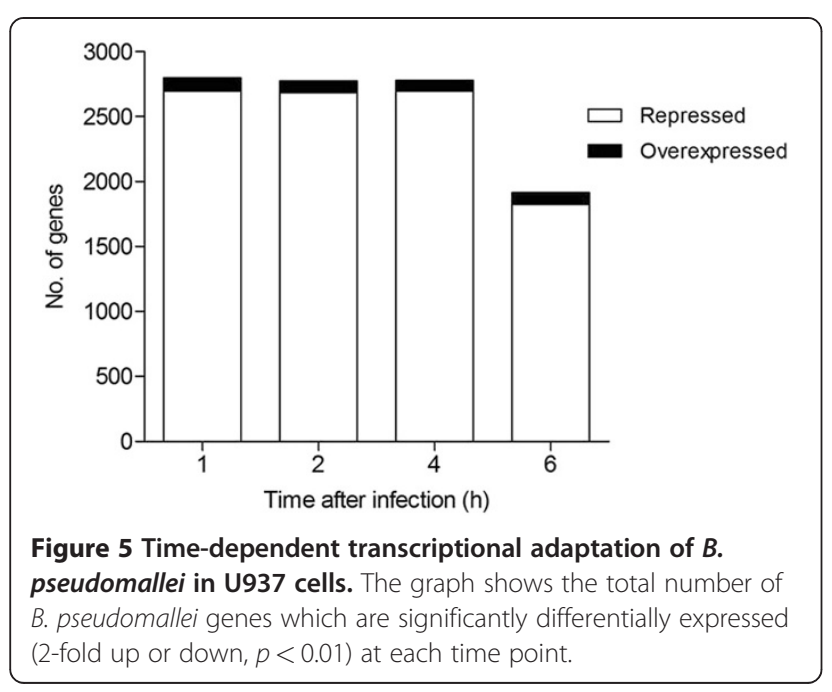

to genes that have some functional information are shown and discussed below. The identified genes are discussed according to functional categories.

\section{Intracellular metabolism and ion transport}

In host-pathogen interactions, sufficient nutrition levels are necessary for the successful survival of the pathogen. Once intracellular, the bacteria must make metabolic adjustments to adapt to changes in nutrient availability. We noted a robust shutdown in the expression of $B$. pseudomallei genes involved in metabolism. Genes involved in glycolysis and oxidative phosphorylation were consistently down-regulated in intracellular $B$. pseudomallei. In addition, most genes involved in energy metabolism such as ATP synthase and NADH dehydrogenase were down-regulated (Figure 4B). Collectively, these data suggest that intracellular B. pseudomallei have lower energy requirements and limit their energy production during the initial stage of infection. Several anaerobic metabolism genes were induced in intracellular B. pseudomallei. Anaerobic metabolism pathway genes such as BPSS1279 (threonine dehydratase), BPSL1771 


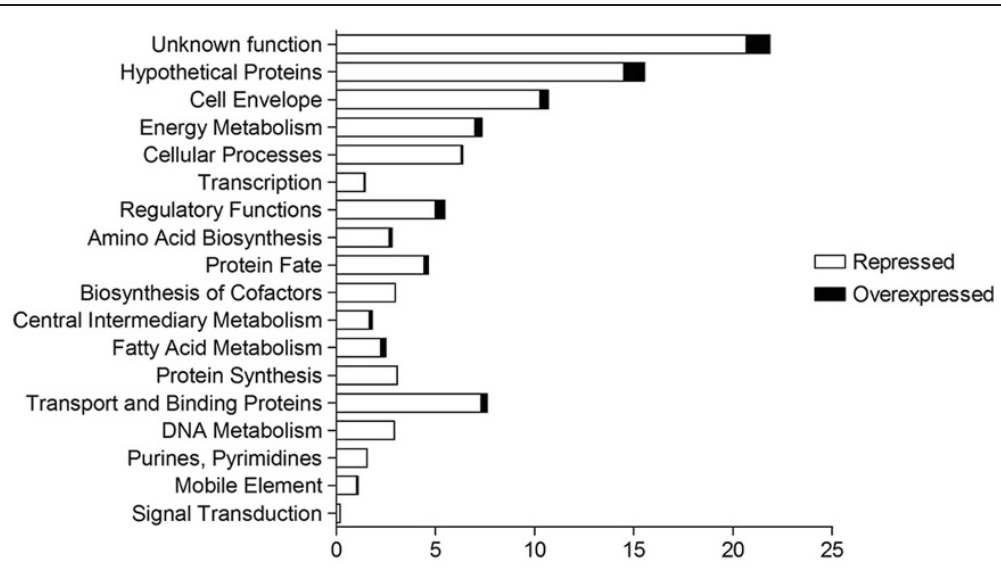

Figure 6 Functional classification of intracellular B. pseudomallei regulated genes. Bars indicate percentages of genes in each group that were significantly regulated at any time point. Genes were divided into functional categories based on Comprehensive Microbial Resources (CMR) annotations.

Table 1 Twenty-five common up-regulated genes of $B$. pseudomallei during intracellular growth in host macrophages relative to in vitro growth

\begin{tabular}{|c|c|c|c|c|c|}
\hline \multirow[t]{2}{*}{ Gene } & \multirow[t]{2}{*}{ Description } & \multicolumn{4}{|c|}{ Fold Change (in vivo/in vitro) at the indicated time (h) } \\
\hline & & 1 & 2 & 4 & 6 \\
\hline BPSL0184 & Putative rod shape-determining protein & 23.83 & 15.31 & 12.69 & 12.06 \\
\hline BPSL0842 & Benzoylformate decarboxylase & 70.27 & 31.78 & 27.64 & 20.64 \\
\hline BPSL0886 & Hypothetical protein & 12.29 & 8.36 & 6.56 & 5.20 \\
\hline BPSL1067 & Hypothetical protein & 8.39 & 5.15 & 6.75 & 6.27 \\
\hline BPSL1771 & Cobalamin biosynthesis protein $\mathrm{CbiG}$ & 25.36 & 16.66 & 15.21 & 15.76 \\
\hline BPSL1817 & Putative lipoprotein & 30.07 & 14.11 & 12.67 & 11.33 \\
\hline BPSL1902 & Hypothetical protein & 37.91 & 18.81 & 12.46 & 8.39 \\
\hline BPSL2759 & Putative short-chain dehydrogenase & 16.54 & 8.31 & 5.93 & 5.61 \\
\hline BPSL2945 & Allantoicase & 27.90 & 9.57 & 8.83 & 7.41 \\
\hline BPSL3354 & Putative cytochrome & 18.66 & 11.70 & 8.64 & 7.62 \\
\hline BPSS0140 & Sugar $A B C$ transport system, lipoprotein & 7.80 & 16.62 & 13.56 & 13.94 \\
\hline BPSS0142* & Sugar ABC transport system, ATP-binding protein & 4.62 & 10.04 & 11.34 & 18.79 \\
\hline BPSS0143 & ROK family transcriptional regulator & 4.78 & 9.97 & 11.80 & 18.64 \\
\hline BPSS0404 & Methylamine utilization protein & 49.46 & 36.31 & 30.14 & 23.42 \\
\hline BPSS0433 & Hypothetical protein & 11.87 & 5.84 & 7.70 & 10.59 \\
\hline BPSS0529 & Lipoprotein & 26.49 & 19.04 & 14.10 & 10.68 \\
\hline BPSS1279 & Threonine dehydratase & 17.47 & 11.84 & 10.80 & 11.03 \\
\hline BPSS1498 * & tssD, type VI secretion system Hcp protein & 7.38 & 93.61 & 130.11 & 181.92 \\
\hline BPSS1499 & tssE, type VI secretion system lysozyme & 4.72 & 58.87 & 99.06 & 160.21 \\
\hline BPSS1505 & tagB, type VI secretion system hypothetical protein & 6.49 & 14.01 & 18.40 & 27.97 \\
\hline BPSS1508 & tssJ, type VI secretion system lipoprotein & 5.04 & 6.27 & 8.17 & 9.88 \\
\hline BPSS1728 * & Secretion/activator protein & 3.00 & 23.60 & 29.33 & 24.54 \\
\hline BPSS1892 & catA, catechol 1,2-dioxygenase & 7.76 & 19.46 & 16.64 & 16.53 \\
\hline BPSS1893 & catC, muconolactone delta-isomerase & 10.57 & 9.30 & 7.33 & 6.30 \\
\hline BPSS2276 & LysR family regulatory protein & 59.21 & 41.83 & 26.33 & 19.45 \\
\hline
\end{tabular}

Note: * Genes selected for real-time qPCR analysis. 
Table 2 Gene function enrichment analysis of B. pseudomallei common up-regulated and down-regulated genes throughout growth within host macrophages

\begin{tabular}{llll}
\hline Functional class or pathway & No. of genes regulated & No. of genes in genome & Significance $(\boldsymbol{p}$-value $)$ \\
\hline Up-regulated genes & 3 & 29 & $3.33 \times 10^{-2}$ \\
Benzoate degradation via hydroxylation & & & 39 \\
Down-regulated genes & 22 & 46 & $7.98 \times 10^{-10}$ \\
Amino sugar and nucleotide sugar metabolism & 23 & 24 & $2.65 \times 10^{-9}$ \\
Bacterial chemotaxis & 13 & 24 & $2.36 \times 10^{-5}$ \\
Lipopolysaccharide biosynthesis & 11 & 38 & $7.93 \times 10^{-4}$ \\
Peptidoglycan biosynthesis & 11 & 32 & $2.72 \times 10^{-2}$ \\
Flagella assembly & 15 & 21 & $2.41 \times 10^{-5}$ \\
Alanine, aspartate and glutamate metabolism & 9 & 104 & $6.33 \times 10^{-3}$ \\
Fatty acid biosynthesis & 30 & 36 & $3.68 \times 10^{-6}$ \\
Two-component system & 11 & 60 & $1.81 \times 10^{-2}$ \\
Glycolysis/gluconeogenesis & 15 & & $1.55 \times 10^{-2}$ \\
Oxidative phosphorylation & & & \\
\hline
\end{tabular}

(cobalamin biosynthesis protein $\mathrm{CbiG}$ ) and BPSS0842 (benzoylformate decarboxylase) were up-regulated throughout the infection period. Nevertheless, none of the components of the anaerobic respiratory chain showed significant changes in expression except for BPSL2311 (putative respiratory nitrate reductase delta chain) and BPSL2312 (putative respiratory nitrate reductase gamma chain) that were induced at the early stage of infection.

Other induced genes were catAC genes, which are involved in benzoate degradation, indicating that intracellular B. pseudomallei utilized aromatic compounds as a source of carbon. Increased expression of phenylacetic acid (PA) pathway genes at the later stage of infection ( $4 \mathrm{~h}$ and $6 \mathrm{~h}$ post-infection) was also observed (Figure 4B). The major nitrogen source in the intracellular compartment is most likely methylamine and purine as suggested by the increased expression of methylamine utilization protein (BPSS0404) and allantoicase (BPSL2945). Allantoicase is involved in purine metabolism and provides a secondary nitrogen source under nitrogen limiting conditions [26].
Expression of virulence and virulence-associated factors We observed the repression of genes encoding proteins that are well characterised as B. pseudomalle $i$ virulence factors. These include the main capsular polysaccharide biosynthesis (BPSL2787-BPSL2810) genes, two potential surface polysaccharide biosynthesis gene clusters (BPSS0417-BPSS0429 and BPSS1825-BPSS1834), majority of genes in the lipopolysaccharide (LPS) biosynthesis cluster and genes encoding for flagella assembly and chemotaxis. We also noted the repression of $b s p R$, a regulator recently shown to control the expression of TT3SS-3 genes [27]. The inhibition of this regulator leads to the reduced expression of T3SS-3 controlled genes in intracellular B. pseudomallei.

One of the six clusters of the type VI secretion system, the tss-5 cluster (BPSS1493-BPSS1511), was upregulated up to 182-fold during intracellular infection (Figure 8). This observation is consistent with previous reports on the induction of three genes in this cluster, tss $H-5$, tssI-5 and tss $M-5$, upon invasion of macrophages [18]. We also observed the induction of genes flanking

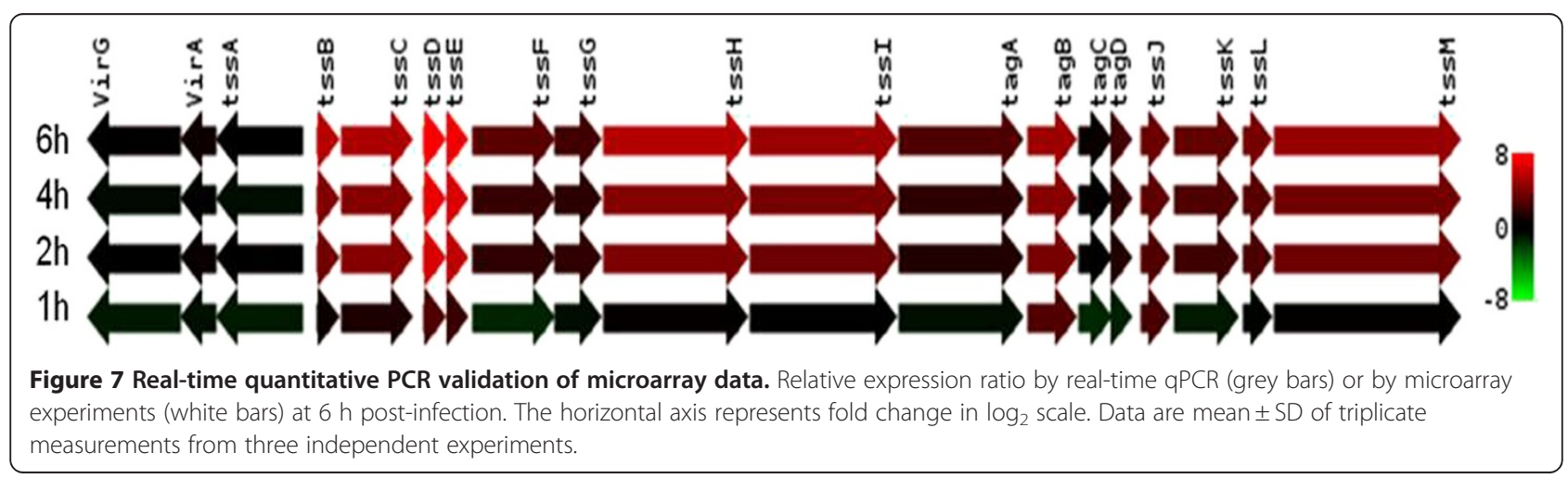




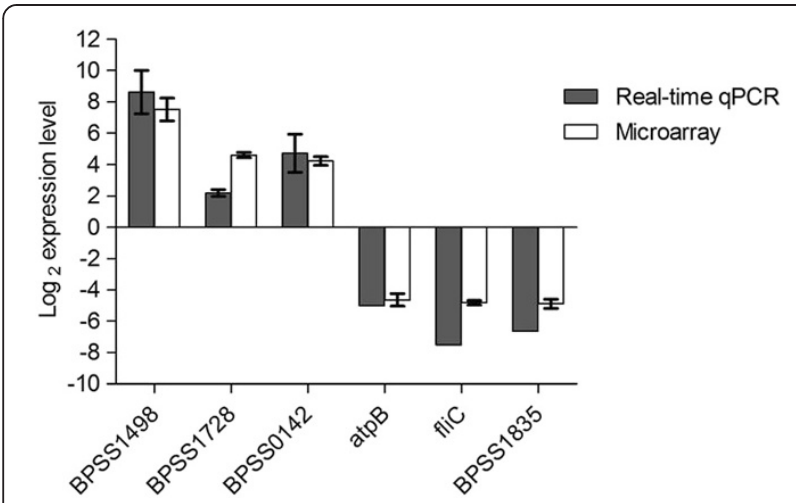

Figure 8 Regulation of the type VI secretion system tss- 5 cluster. The heat-map shows the expression ratio of each gene in the T6SS tss-5 cluster during intracellular growth in host macrophages relative to in vitro growth. Expression values are determined from the SAM analysis with red representing upregulation (ratio of +8.0 ) and green representing down-regulation (ratio of -8.0 ) on a $\log _{2}$ scale.

the tss-5 cluster, bimA (Burkholderia intracellular motility A)(BPSS1492) and BPSS1512 at 2 to $6 \mathrm{~h}$ postinfection. Moreover, the hemolysin activator-like protein precursor, fhaC (BPSS1728) gene was significantly upregulated during intracellular infection. Consistently, the large filamentous hemagglutinin precursor, fhaB (BPSS1727) gene, a potential virulence factor of B. pseudomallei [20], was induced between 2 to $6 \mathrm{~h}$ postinfection.

\section{Stress responses genes}

The general stress-responsive alternative sigma factor rpoS transcribes genes involved in bacterial survival under conditions of environmental stress. In B. pseudomallei, rpoS is a regulator of carbon starvation and oxidative stress [28].We observed down-regulation of rpoS throughout intracellular growth. Furthermore, the expression of most of the genes encoding sigma factors was repressed in the intracellular bacteria. Under oxidative stress, rpoS regulates $o x y R$ and katG-dpsA operons [29]. As expected, we observed down regulation of $\operatorname{oxy} R$ and $k a t G-d p s A$ expression at 1 and $2 \mathrm{~h}$ post-infection. The expression of other genes in the oxy $R$ regulon was either repressed or did not change significantly relative to control cells. Moreover, class I stress response genes including dnaJ, dnaK, $h r c A$ and groEL were not significantly regulated, except for groES, which was induced at $6 \mathrm{~h}$ post-infection.

\section{DNA topology and growth arrest within macrophages}

Modification of DNA topology plays a major role in assisting DNA replication and protein synthesis. Prokaryotic DNA is usually maintained in a negatively supercoiled form by topoisomerases and the level of supercoiling can be affected by several environmental parameters [30]. The ability of DNA gyrase to generate negative supercoils in DNA is inhibited when ATP levels are reduced [31]. In this study, DNA topoisomerase IV subunit A (parC), DNA topoisomerase IV subunit B (parB) and DNA gyrase subunit A (gray) genes were down-regulated. The repression of gyrase suggests a relaxation in bacterial DNA supercoiling during B. pseudomallei intracellular growth in U937. Additionally, the nucleotide-associated regulator protein Fis was also negatively regulated. Fis expression depends on superhelical density whereby maximum density is observed at high levels of negative supercoiling [32].

We found that bacterial genes involved in cell division (ftsABH) were down-regulated indicating reduced cell division activity. The repression of $\min D$ and $\min E$ genes, which mediate spatial regulation of cytokinesis in bacteria [33], and chromosome partitioning genes, parA and $\operatorname{par} B$ was also observed. Furthermore, replicative DNA helicase dnaB, DNA polymerase III subunit alpha $d n a E$ and DNA polymerase III subunit chi genes were also down-regulated. Consistent with the down regulation of catabolic and cell replication genes, the expression of RNA polymerase (rpoABCZ) and ribosomal subunit genes (S1, S10, S21, L11 and L28) was also reduced throughout the infection period. Collectively, these support the view that cell division and replication processes are interrupted during infection, leading to slower growth kinetics of intracellular B. pseudomallei.

\section{Discussion}

Numerous studies have been conducted on the cellular interaction between B. pseudomallei and eukaryotic cells, especially the ability of this pathogen to survive within host cells. In this study, we investigated the global B. pseudomallei transcriptome profile during early stage infection of human monocyte-like U937 cells. Initially, we demonstrated that replication kinetics of intracellular B. pseudomallei within U937 cells is similar to data from a number of previous studies [8,34]. The bacteria were able to survive in macrophage cells, albeit, at a slower growth rate compared to bacteria grown in RPMI. Reduced bacterial growth kinetics during infection was also reported in a hamster model of melioidosis [35]. Furthermore, this reduced bacterial replication kinetics in the intracellular environment in comparison with in vitro growth is not limited to B. pseudomallei [3638]. B. pseudomallei infection was also cytotoxic to macrophage cells with about $20 \%$ cell mortality at $24 \mathrm{~h}$ post infection which, to some extent, explains the reduced number of recovered bacteria with prolonged incubation. Electron micrographs of infected U937 cells revealed disruption of internal structures and formation of oncotic cells. The cytotoxic and cytopathy effects of 
B. pseudomallei infection in our study are in agreement with Sun et al. [21], who reported high cytotoxicity (40$70 \%)$ and oncotic death in infected THP-1 cells. We also observed the loss of phagosome membrane in infected macrophages as early as $2 \mathrm{~h}$ post-infection resulting in the presence of free replicating bacilli in the cytoplasm at later time points.

The ability to survive within and lyse macrophage cells most probably contributes to the spread of this bacterium and disease progression. We attempted to identify mechanisms that underlie the adaptive ability of B. pseudomallei during infection by comparing intracellular bacteria transcripts to that of in vitro grown bacteria. During infection, we observed that intracellular B. pseudomallei demonstrated a lower energy requirement and production by shutting down metabolic activity. The reduced expression of catabolic and house-keeping genes is consistent with the reduced growth rate observed for intracellular B. pseudomallei. The induction of anaerobic metabolism pathway genes is suggestive of limited oxygen concentrations in the cytosol although expression of genes of the anaerobic respiratory chain was not significantly altered. The importance of this finding requires further investigation.

B. pseudomallei utilizes alternative metabolites during infection [35]. Our study suggests that B. pseudomallei utilize aromatic carbon compounds such as benzoate and PA as carbon sources for intracellular survival. PA can inhibit the inducible nitric oxide synthase (iNOS) and LPS-induced expression of cytokines in rat primary astrocytes, microglia and macrophages [39]. Moreover, PA can repress DNA binding and transcriptional activities of $\mathrm{NF}_{\mathrm{B}} \mathrm{B}$, an important upstream modulator for cytokine and iNOS expression in macrophages [40]. The up-regulation of PA catabolic pathway genes in intracellular bacteria might partly explain why macrophages infected with $B$. pseudomallei failed to activate the production of iNOS [41]. The putative B. cenocepacia ring PA-coenzyme A hydroxylation system was found to be essential for full pathogenicity in infected Caenorhabditis elegans [42].

There are three T3SSs present in B. pseudomallei and only T3SS-3 is involved in virulence [43]. B. pseudomallei T3SS-3 shows high homology to the Inv/Mxi-Spa type III secretion systems of Salmonella and Shigella [13]. As T3SS-3 is known to be essential in facilitating invasion and early phagosomal escape into the cytosol $[13,14]$, the down-regulation observed in our study is consistent with this function as it would be conceivable that T3SS-3 is important at the beginning of intracellular life during invasion, but not important at the later stages of intracellular infection. Similarly, the role of T6SS-1 is reported to be crucial during the transition from phagosome to cytosol [15]. Thus, it is possible that the induction of T6SS-1 in this study plays a major function in ensuring pathogen survival and replication in the cytosol. In B. mallei, a close related species of B. pseudomallei, an effector protein of T6SS (BMAA0742) was recognized by glanders antiserum, indicating production of this protein in vivo during infection [44]. In this study, high induction of tssD-5 (BPSS1498), an effector Hcp1 protein of T6SS was observed throughout the infection period. A recent study on B. pseudomallei T6SS Hcp proteins has shown that $t s s D-5$ deletion mutant was attenuated in a hamster infection model, exhibited growth defects and was only weakly cytotoxic to RAW264.7 macrophages [45]. Additionally, human melioidosis serum samples were found to react with Hcp1 protein, consistent with our observation (data not shown). These results suggest that Hcp1 is produced by B. pseudomallei in vivo during infection, is immunogenic and vital for intracellular survival of $B$. pseudomallei.

The genes $\operatorname{bim} A$, fhaB and fhaC are known proteins that mediate actin tail formation involved in cell-to-cell spread. In this study, expression of these three genes is up-regulated. The over expression of BimA has previously been shown in intracellular B. pseudomallei and mutation of $\operatorname{bim} A$ abolished actin-based motility of this pathogen in J774.2 cell [46]. In Bordetella pertussis, the FhaC dependent filamentous hemagglutinin (FHA) facilitates attachment to the host cell during infection [47] and initiates killing of macrophages to avoid the host cell mediated immune response whilst infection is being established [48]. Through a genome-wide function screen of B. pseudomallei strain K96243, both fhaB and fha $C$ were found to form part of the anti-macrophage loci and contribute to the formation of dramatic actin projections extending towards neighbouring cells [49].

Interestingly, in our study, well-characterized virulence genes of B. pseudomallei such as capsular polysaccharide, LPS and flagella were either repressed or not significantly expressed. Similar profiles were noted in a hamster model, wherein most of the genes encoding for capsule, LPS and flagella biosynthesis were either downregulated or not significantly changed in expression [35]. This could be due to the equal expression of these genes under in vivo and in vitro conditions concomitant with previous evidence that capsule, LPS, flagellum and other virulent determinants are constitutively produced in vitro [35]. A recent report of a similar study on macrophages infected by $B$. cenocepacia demonstrated that this intracellular pathogen's ability to adapt and respond to the intracellular milieu was not dependent on the expression of any specific virulence-associated factors [50]. Hence, this ability to adapt to various niches appears to be conserved amongst the soil-derived Burkholderia spp. clinical pathogens. Down regulation of flagella genes and subsequently the absence of flagellin, 
may also reflect the bacteria's attempt to limit immune recognition especially by toll-like receptors by modulating surface structures. In Gram negative bacteria, flagellin is recognized by TLR 5 which triggers the secretion of various cytokines and chemokines, leading to an inflammatory response [51]. By dampening the activation of TLR5, the innate immune response towards B. pseudomallei is reduced and bacterial clearance is prevented. Consistent with our observations, B. pseudomallei isolates failed to significantly increase TLR5 expression in both cell culture [52] and BALB/c mice infection models [53]. Nanagara et al. [54] also suggested that the suppression of $B$. pseudomallei surface antigens in naturally infected human synovial tissues aids in bacteria survival against host immune responses and antibiotic treatment.

Intracellular bacteria are constantly exposed to a range of stresses such as oxidative burst and formation of oxygen radicals by the host cellular defence mechanisms. Several in vitro studies designed to mimic the host environment have identified the expression of the $d s p A$ [55] and $\operatorname{ahpC-}$ $k a t G$ [56] genes as necessary for survival and growth under oxidative stress. Surprisingly, in our study, expression of the majority of oxidative stress related genes was repressed or not significantly altered. This observation suggests the ability of $B$. pseudomallei to rapidly evade oxidative stress by escaping from the phagosome [22]. This enables the bacteria to enter the cytoplasm, a more favourable environment for survival and growth where nutrients are freely available and microbicides do not operate [7].

\section{Conclusions}

This is the first report of a complete transcriptome profile of intracellular B. pseudomallei within macrophages. We have determined that B. pseudomallei adapts rapidly to the intracellular environment through the regulation of bacteria metabolism and growth rate and the possibility of host cell immune response avoidance through shutdown of known virulence factors. Proteins encoded by genes induced during infection including genes encoding the T6SS cluster are potential diagnostic candidates or targets for anti-microbial development.

\section{Methods}

\section{Bacterial strain and growth conditions}

B. pseudomallei D286, a clinical isolate previously described by Lee [57], was grown on Ashdown agar for $48 \mathrm{~h}$ or in Luria-Bertani (LB) broth overnight at $37^{\circ} \mathrm{C}$. Prior to infection, overnight cultures were diluted to 1:50 in LB broth and grown to mid-logarithmic phase $\left(\mathrm{OD}_{600}=0.4-0.6\right)$ at $37{ }^{\circ} \mathrm{C}, 250 \mathrm{rpm}$ for $3 \mathrm{~h}$.

\section{Cell culture and infection model}

Human monocyte-like U937 cells (CRL-1593.2) were maintained in RPMI1640 medium (Gibco) supplemented with $10 \%$ fetal bovine serum (Hyclone), $2 \mathrm{mM} \mathrm{L-}$ glutamine, $10 \mathrm{mM}$ HEPES and $1 \mathrm{mM}$ sodium pyruvate (Invitrogen). For infection assays, $2 \times 10^{5}$ cells/well were seeded in 12-well cell culture plates, while for RNA extraction, approximately $3 \times 10^{7}$ cells/flask were seeded in T175 flasks. U937 cells were supplemented with $10 \mathrm{ng} /$ $\mathrm{ml}$ phorbol myristate acetate (PMA) (Sigma) to induce macrophage differentiation $48 \mathrm{~h}$ prior to infection [58]. Induced U937 cells were washed once with Hanks' Balance Salt Solution (Gibco) to remove traces of PMA before addition of bacteria at a multiplicity of infection of 10. After $2 \mathrm{~h}$ at $37{ }^{\circ} \mathrm{C}$, extracellular bacteria were removed by extensive washing with PBS. Fresh media containing $250 \mu \mathrm{g} / \mathrm{ml}$ kanamycin was added to each plate or flask and infected cells were incubated at $37{ }^{\circ} \mathrm{C}$ until lysed.

\section{Intracellular survival and eukaryotic cell viability determination}

Intracellular survival of B. pseudomallei in U937 was estimated as previously described with some modifications [8]. At different time points following the initial $2 \mathrm{~h}$ infection with B. pseudomallei, infected macrophage monolayers were washed three times with PBS and intracellular $B$. pseudomallei were harvested by adding $500 \mu \mathrm{l}$ of $1 \%$ saponin in PBS to each well. After $5 \mathrm{~min}$ incubation at $37^{\circ} \mathrm{C}$, cell lysates were collected and serially diluted 10-fold in PBS and aliquots were plated onto Ashdown agar to assess viable bacterial counts.

To determine the overall viability of macrophage cells following bacterial infection, trypan blue exclusion was used. At selected time points after infection with bacteria, the monolayers were washed three times with PBS and gently scrapped off from the wells. Trypan blue solution was added to cell suspensions and stained infected cells were visualized under an inverted microscope. Assays were performed in triplicate and repeated at least three times. The number of intact viable cells was expressed as a percentage relative to viable uninfected cells.

\section{Transmission electron microscopy}

Transmission electron microscopy for infected U937 cells was performed as previously described [22] with some modifications. Briefly, cells were fixed for $24 \mathrm{~h}$ in $4 \%$ glutaraldehyde at $4{ }^{\circ} \mathrm{C}$ and washed three times in PBS. After a secondary fix for $2 \mathrm{~h}$ in $1 \%$ osmium tetroxide at $4{ }^{\circ} \mathrm{C}$, the specimens were washed three times in PBS. The specimens were then dehydrated in a graded series of acetone/water containing $30 \%$ to $100 \%$ acetone. The specimens were infiltrated with acetone-resin mixture, embedded into resin hard mix and left to polymerize at $60{ }^{\circ} \mathrm{C}$ for 24 to $48 \mathrm{~h}$. Ultrathin sections 
were stained with lead citrate and viewed on a Hitachi $\mathrm{H}-7100$ transmission electron microscope.

\section{RNA extraction}

At each time point (1, 2, 4 and $6 \mathrm{~h}$ post-infection), macrophage monolayers were washed and infected macrophages from three T175 flasks were combined and lysed in $1 \%$ saponin in PBS (sterile and filtered) for $5 \mathrm{~min}$ at $37^{\circ} \mathrm{C}$ [25]. Lysates were collected and subjected to differential centrifugation; first at $800 \times g$ for $5 \mathrm{~min}$ to sediment eukaryotic cells and cellular debris, and secondly at $8,000 \times g$ for $10 \mathrm{~min}$ to pellet bacterial cells. Pellets were immediately snap-frozen in liquid nitrogen and kept at $-80{ }^{\circ} \mathrm{C}$ until extraction. Bacterial RNA was prepared using the Qiagen's RNeasy Mini Kit and oncolumn DNase I digestion was performed. Total RNA obtained was further purified by ethanol/ammonium acetate precipitation. Control RNA from in vitro grown bacteria was obtained by diluting overnight cultures and growing stationary at $37{ }^{\circ} \mathrm{C}$ to mid-logarithmic phase in complete RPMI1640 medium under $5 \% \mathrm{CO}_{2}$. These conditions mimicked those used for the cell infection experiments. Control bacteria were treated similarly and RNA was isolated as described above. Control U937 RNA was isolated using Trizol and purified with the RNeasy Mini Kit. The concentration, quality and integrity of all RNA isolated were analysed using the Nanodrop ${ }^{\circledR}$ ND-1000 and Agilent 2100 Bioanalyser.

A preliminary control experiment demonstrated that incubation in $1 \%$ saponin for $5 \mathrm{~min}$ at $37{ }^{\circ} \mathrm{C}$ did not affect the viability of $B$. pseudomallei (data not shown). Additionally, when comparing the transcriptional profile of $B$. pseudomallei before and after treatment with saponin and differential centrifugation, we found that these treatments caused no significant changes in bacterial gene expression (data not shown), consistent with studies on other bacterial pathogens [24,59].

\section{Construction of $B$. pseudomallei DNA microarrays}

Microarray containing probes to the annotated ORFs of the B. pseudomallei reference strain K96243 was designed using Agilent's eArray 5.0 web-based tool and synthesized using Agilent's 60-mer Sure Print technology. Probes were filtered using a perfect match filter to eliminate probes with identical sequences and a similarity score filter to discard probes with significant similarity to other parts of the target genome. Quality control of the probes was also done based on their base composition (BC) score. BC score is a numerical value that defines the quality of the probe based upon its base composition and distribution, with $\mathrm{BC} \_1$ being the best and $\mathrm{BC}$ _Poor the worst. Filtered probes with higher $\mathrm{BC}$ scores, namely BC_1 and BC_2, were included in the array, while probes with lower BC scores, such as BC_3 and $\mathrm{BC}_{-} 4$, were excluded. A total of 5,721 probe sequences passed the filtering and quality control criteria and were replicated and randomly distributed in a microarray of 15,000 probes to fit Agilent $8 \times 15 \mathrm{~K}$ microarray format. In summary, the B. pseudomallei $8 \times 15$ K microarrays (GEO reference GPL13233) allowed for the analysis of 5,721 non-cross-hybridizing ORFs of B. pseudomallei K96243.

\section{Sample labelling and hybridization}

Prior to labelling, bacterial RNA was polyadenylated and reverse transcribed. Polyadenylation of bacterial RNA was based on the PAP method using A-plus ${ }^{\mathrm{TM}}$ Poly(A) Polymerase Tailing kit (Epicentre). Poly-a tailing was carried out to provide a priming site for the synthesis of first strand cDNA. Polyadenylation was terminated by ethanol/ammonium acetate precipitation. cDNA synthesis, labelling and hybridization were done according to the Agilent one-colour microarray protocols (available at http://www.chem.agilent.com/Library/usermanuals/Public/G4140-90040_GeneExpression_One-color_v6.5.pdf).

Washes were also conducted according to standard Agilent protocols. An additional wash with acetonitrile was conducted to completely dry the arrays. Arrays were scanned with the Agilent Technologies Scanner model G2505B. Spot intensities and other quality control features were extracted with Agilent's Feature Extraction Software version 9.5.3.1. In this study, 3 independent hybridizations using RNA samples isolated from 3 separate assays (biological replicates) were performed for each incubation time point and control bacteria.

\section{Data analysis}

Microarray quality was assessed through the use of Agilent's control features and only arrays that passed the recommended criteria were included in the analysis. Processed signals obtained from Feature Extraction were used as signal intensities for analysis. The data was filtered using a signal to noise ratio criteria and only features for which the background-subtracted signal was 2.6 times above the background standard deviation for that feature in at least 13 of the 15 arrays, were retained. A total of 5,391 genes passed the filtering process. Arrays were median normalized with BRB-ArrayTools (http://linus.nci.nih.gov) to adjust the scale of intensities across samples and arrays. Filtered and normalized data were subjected to Significance Analysis of Microarray (SAM) analysis in which a two class unpaired analysis was performed and genes with a false discovery rate $($ FDR $)<0.01$ and fold change $\geq 2$ were defined as significantly differentially expressed. Hierarchical clustering analysis with Euclidean correlation was performed using TIGR-MeV software version 4.3.2 (www.tigr.org). Functional classifications were carried out based on 
Table 3 List of oligonucleotides used in real-time qPCR experiments

\begin{tabular}{|c|c|c|}
\hline Gene & Primer name & Nucleotide sequence $\left(5^{\prime}-3^{\prime}\right)$ \\
\hline \multirow[t]{2}{*}{$c y d B$} & CydB_F cydB_R & GATCCGAAGAGCAGCC \\
\hline & & CAGCCCGTGTAGAGCAG \\
\hline \multirow[t]{2}{*}{ BPSS0142 } & BPSS0142_F & ACCGATAACCTGTTCCG \\
\hline & BPSS0142_R & CGTAGATTTCCGCCATC \\
\hline \multirow[t]{2}{*}{ BPSS1498 } & BPSS1498_F & TCAAGGTCAAAGGAAAAAC \\
\hline & BPSS1498_R & AAGGCGAGGATGTGGAT \\
\hline \multirow[t]{2}{*}{ BPSS1728 } & BPSS1728_F & AGAGCCGCCAAGATCAA \\
\hline & BPSS1728_R & GCCGAGACCCGAGTTAT \\
\hline \multirow[t]{2}{*}{$\operatorname{atp} B$} & $a t p B \_F a t p B \_R$ & GTGGCTITAACGATATGGC \\
\hline & & TGATGCGAGGTGGAGAA \\
\hline \multirow[t]{2}{*}{ flic } & flic_F flic_R & CAGACGAACTACAACGGC \\
\hline & & ATGCTITGCGTGAGGTC \\
\hline BPSS1835 & BPSS1835_F BPSS1835_R & CGTGAAGAAAATCGTCG GAGTCGTAATGTCCCCAC \\
\hline
\end{tabular}

Comprehensive Microbial Resources (CMR) annotations (www.cmr.jcvi.org). Gene function enrichment analysis was performed on the DAVID 6.7 database (http://david. abcc.ncifcrf.gov/home.jsp) [60] using a Fisher Exact test with Benjamini and Hochberg multiple testing correction $(p<0.05)$. All microarray results have been deposited in the GEO database (http://www.ncbi.nlm.nih.gov/ geo/) with the GEO series accession number GSE27558.

\section{Real-time quantitative PCR}

Total RNA was treated with DNase I (Invitrogen) to remove any traces of DNA and converted to cDNA using Superscript III (Invitrogen) with random hexamers, according to the manufacturer's instructions. Real-time quantitative PCR was performed on $10 \mathrm{ng}$ cDNA in a final volume of $20 \mu \mathrm{l}$ in a SDS7500 with Power Sybr Green PCR Master Mix (Applied Biosystems). All experiments were conducted three times, which yielded 9 measurements per gene (representing 3 technical replicates of 3 biological replicates) and the relative expression ratios were calculated using REST-MCS [61]. The gene coding for cytochrome d ubiquinol oxidase subunit II, $c y d B$, was used as the reference gene for normalization. $C y d B$ was chosen as the reference gene because it did not show any significant changes in expression in the microarray experiment. Primers are described in Table 3. The correlation between expression ratios obtained from the microarray and real-time qPCR was evaluated with Pearson correlation (Microsoft Office Excel).

\section{Additional file}

Additional file 1: List of 1259 common down-regulated genes of $B$. pseudomallei during intracellular growth in host macrophages relative to in vitro growth.

\section{Competing interests}

The authors declare that they have no competing interests

\section{Authors' contributions}

SC contributed to the design of the study, performed all the experiments and data analysis and wrote the manuscript. LC contributed to the microarray interpretation and analysis and helped in performing real-time quantitative PCR. SN conceived and coordinated the study, contributed to the experimental design and wrote the manuscript. All authors read and approved the final manuscript.

\section{Acknowledgements}

This project was funded by the Ministry of Science, Technology and Innovation of Malaysia under the R\&D Initiatives Grant Program awarded to S.N. We are grateful to the RNA Biology Laboratory and the Microarray Facility of the University of Aveiro for assistance in microarray data analysis, and to the Electron Microscopy Unit of Universiti Kebangsaan Malaysia and Universiti Putra Malaysia for assistance in electron microscopy.

\section{Author details}

${ }^{1}$ School of Biosciences and Biotechnology, Faculty of Science and Technology, Universiti Kebangsaan Malaysia, Bangi 43600, Malaysia. ${ }^{2}$ CESAM and Departamento de Biologia, Universidade de Aveiro, Campus de Santiago, Aveiro 3810-193, Portugal.

Received: 18 October 2011 Accepted: 23 July 2012 Published: 23 July 2012

\section{References}

1. White NJ: Melioidosis. Lancet 2003, 361:1715-1722.

2. Brett PJ, Woods DE: Pathogenesis of and immunity to melioidosis. Acta Trop 2000, 74:201-210.

3. Cheng AC, Currie BJ: Melioidosis: epidemiology, pathophysiology, and management. ClinMicrobiol Rev 2005, 18(2):383-416.

4. Currie BJ, Fisher DA, Anstey NM, Jacups SP: Melioidosis: acute and chronic disease, relapse and re-activation. Trans R Soc Trop Med Hyg 2000, 94:301-304.

5. Jenney AW, Lum G, Fisher DA, Currie BJ: Antibiotic susceptibility of Burkholderia pseudomallei from tropical northern Australia and implications for therapy of melioidosis. Int J Antimicrob Agents 2001, 17 (2):109-113.

6. Wiersinga WJ, van der Poll T, White NJ, Day NP, Peacock SJ: Melioidosis: insights into the pathogenicity of Burkholderia pseudomallei. Nat Rev Microbiol 2006, 4(4):272-282. 
7. Harley VS, Dance DAB, Tovey G, McCrossan MV, Drasar BS: An ultrastructural study of the phagocytosis of Burkholderia pseudomallei. Microbios 1998, 94:35-45

8. Jones AL, Beveridge TJ, Woods DE: Intracellular survival of Burkholderia pseudomallei. Infect Immun 1996, 64(3):782-790.

9. Inglis TJJ, Rigby P, Robertson TA, Dutton NS, Henderson M, Chang BJ: Interaction between Burkholderia pseudomallei and Acanthamoeba species results in coiling phagocytosis, endamebic bacterial survival, and escape. Infect Immun 2000, 68(3):1681-1686.

10. Stevens MP, Friebel A, Taylor LA, Wood MW, Brown PJ, Hardt WD, Galyov EE: A Burkholderia pseudomallei type III secreted protein, BopE, facilitates bacterial invasion of epithelial cells and exhibits guanine nucleotide exchange factor activity. J Bacteriol 2003, 185(16):4992-4996.

11. Kespichayawattana W, Rattanachetkul S, Wanun T, Utaisincharoen P Sirisinha S: Burkholderia pseudomallei induces cell fusion and actinassociated membrane protrusion: a possible mechanism for cell-to-cell spreading. Infect Immun 2000, 68(9):5377-5384.

12. Stevens MP, Galyov EE: Exploitation of host cells by Burkholderia pseudomallei. Int J Med Microbiol 2004, 293:549-555.

13. Stevens MP, Wood MW, Taylor LA, Monaghan P, Hawes P, Jones PW, Wallis TS, Gaylov EE: An Inv/Mxi-Spa like type III protein secretion system in Burkholderia pseudomallei modulates intracellular behaviour of the pathogen. Mol Microbiol 2002, 46(9):649-659.

14. Suparak S, Kespichayawattana W, Haque A, Easton A, Damnin S, Lertmemongkolchai G, Bancroft GJ, Korbsrisate S: Multinucleated giant cell formation and apoptosis in infected host cells is mediated by Burkholderia pseudomallei type III secretion protein BipB. J Bacteriol 2005, 187(18):6556-6560.

15. Chen Y, Wong J, Sun GW, Liu Y, Tan GG, Gan Y: Regulation of type VI secretion system during Burkholderia pseudomallei infection. Infect Immun 2011, 79(8):3064-3073.

16. Ma AT, McAuley S, Pukatzki S, Mekalanos JJ: Translocation of a Vibrio cholerae type $\mathrm{VI}$ secretion effector requires bacterial endocytosis by host cells. Cell Host Microbe 2009, 5(3):234-243.

17. Mougous JD, Cuff ME, Raunser S, Shen A, Zhou M, Gifford CA, Goodman AL, Joachimiak G, Ordoñez CL, Lory S, Walz T, Joachimiak A, Mekalanos JJ: A virulence locus of Pseudomonas aeruginosa encodes a protein secretion apparatus. Science 2006, 312:1526-1530.

18. Shalom G, Shaw JG, Thomas MS: In vivo expression technology identifies a type VI secretion system locus in Burkholderia pseudomallei that is induced upon invasion of macrophages. Microbiology 2007, 153:2689-2699.

19. Pilatz S, Breitbach K, Hein N, Fehlhaber B, Schulze J, Brenneke B, Eberl L, Steinmetz I: Identification of Burkholderia pseudomallei genes required for the intracellular life cycle and in vivo virulence. Infect Immun 2006, 74 (6):3576-3586

20. Holden MTG, Titball RW, Peacock SJ, Cerdeño-Tárraga AM, Atkins T, Crossman LC, Pitt T, Churcher C, Mungall K, Bentley SD, Sebaihia M, Thomson NR, Bason N, Beacham IR, Brooks K, Brown KA, Brown NF, Challis GL, Cherevach I, Chillingworth T, Cronin A, Crossett B, Davis P, DeShazer D, Feltwell T, Fraser A, Hance Z, Hauser H, Holroyd S, Jagels K, Keith KE, Maddison M, Moule S, Price C, Quail MA, Rabbinowitsch E, Rutherford K, Sanders M, Simmonds M, Songsivilai S, Stevens K, Tumapa S, Vesaratchavest M, Whitehead S, Yeats C, Barrell BG, Oyston PCF, Parkhill J: Genomic plasticity of the causative agent of melioidosis, Burkholderia pseudomallei. Proc Natl Acad Sci USA 2004, 101(39):14240-14245.

21. Sun GW, Lu J, Pervaiz S, Cao WP, Gan YH: Caspase-1 dependent macrophage death induced by Burkholderia pseudomallei. Cell Microbiol 2005, 7(10):1447-1458.

22. Harley VS, Dance DAB, Drasar BS, Tovey G: Effects of Burkholderia pseudomallei and other Burkholderia species on eukaryotic cells in tissue culture. Microbios 1998, 96:71-93.

23. Waddell SJ, Burcher PD, Stoker NG: RNA profiling in host-pathogen interactions. Curr Opin Microbiol 2007, 10:297-302.

24. Du Y, Lenz J, Arvidson CG: Global gene expression and the role of sigma factors in Neisseria gonorrhoeae in interactions with epithelial cells. Infect Immun 2005, 73(8):4834-4845.

25. Grifantini R, Bartolini E, Muzzi A, Draghi M, Fringimelica E, Berger J, Ratti G, Petracca R, Galli G, Agnusdei M, Giuliani MM, Santini L, Brunelli B, Tettelin H, Rappuoli R, Randazzo F, Grandi G: Previously unrecognised vaccine candidates against group B meningococcus identified by DNA microarrays. Nat Biotechnol 2002, 20:914-921.
26. Trijbels F, Vogels GD: Allantoicase and ureidoglycolase in Pseudomonas and Penicillium species. BiochimBipphysActa 1966, 118(2):387-395.

27. Sun GW, Chen Y, Liu Y, Tan GYG, Ong C, Tan P, Gan YH: Identification of a regulatory cascade controlling type III secretion system 3 gene expression in Burkholderia pseudomallei. Mol Microbiol 2010, 76(3):677-689

28. Subsin B, Thomas MS, Katzenmeier G, Shaw JG, Tungpradabkul S, Kunakorn $M$ : Role of the stationary growth phase sigma factor RpoS of Burkholderia pseudomallei in response to physiological stress conditions. J Bacteriol 2003, 185(23):7008-7014.

29. Jangiam W, Loprasert S, Tungpradabkul S: Role of Burkholderia pseudomallei RpoS in regulation of catalase activities under hydrogen peroxide induction. Science Asia 2008, 34(1):23-29.

30. Dorman CJ: DNA supercoiling and environmental regulation of gene expression in pathogenic bacteria. Infect Immun 1991, 59(3):745-749.

31. Drlica K: Control of bacterial DNA supercoiling. Mol Microbiol 1992, 6 (4):425-433.

32. Schneider $R$, Travers A: The expression of the Escherichia coli fis gene is strongly dependent on the superhelical density of DNA. Mol Microbiol 2000, 38(1):167-175.

33. Rothfield LI, Shih YL, King G: Polar explorers: membrane proteins that determine division site placement. Cell 2001, 106(1):13-16.

34. Wand ME, Muller CM, Titball RW, Michell SL: Macrophage and Galleria mellonela infection models reflect the virulence of naturally occurring isolates of $B$. pseudomallei, B. thailandensis and B. oklahomensis. BMC Microbiol 2011, 11:11.

35. Tuanyok A, Tom M, Dunbar J, Woods DE: Genome-wide expression analysis of Burkholderia pseudomallei infection in a hamster model of acute melioidosis. Infect Immun 2006, 74(10):5465-5476.

36. Boyce JD, Wilkie I, Harper M, Paustian ML, Kapur V, Adler B: Genomic scale analysis of Pasteurella multocida gene expression during growth within natural chicken host. Infect Immun 2002, 70(12):6871-6879.

37. Lucchini S, Liu H, Jin Q, Hinton JCD, Yu J: Transcriptional adaptation of Shigella flexneri during infection of macrophages and epithelial cells: insights into the strategies of a cytosolic bacterial pathogen. Infect Immun 2005, 73(1):88-102.

38. Chatterjee SS, Hossain H, Otten S, Kuenne C, Kuchmina K, Machata S, Domann E, Chakraborty T, Hain T: Intracellular gene expression profile of Listeria monocytogenes. Infect Immun 2006, 74(2):1323-1338.

39. Pahan K, Sheikh FG, Namboodiri AM, Singh I: Lovastatin and phenyl acetate inhibit the induction of nitric oxide synthase and cytokines in rat primary astrocytes, microglia, and macrophages. J Clin Investig 1997, 100:2671-2679.

40. Park JS, Lee EJ, Lee JC, Kim WK, Kim HS: Anti-inflammatory effects of short chain fatty acids in IFN-gamma-stimulated RAW 264.7 murine macrophage cells: involvement of NF-kappaB and ERK signaling pathways. Int Immunopharmacol 2007, 7:70-77.

41. Utaisincharoen P, Tangthawornchaikul N, Kespichayawattana W, Chaisuriya $P$, Sirisinha S: Burkholderia pseudomallei interferes with inducible nitric oxide synthase (iNOS) production: a possible mechanism of evading macrophage killing. Microbiol Immunol 2001, 45(4):307-313.

42. Law RJ, Hamlin JNR, Sivro A, McCorrister SJ, Cardama GA, Cardona ST: A functional phenylacetic acid catabolic pathway is required for full pathogenicity of Burkholderia cenocepacia in the Caenorhabditis elegans host model. J Bacterio/ 2008, 190(21):7209-7218.

43. Warawa J, Woods DE: Type III secretion system cluster 3 is required for maximum virulence of Burkholderia pseudomallei in a hamster infection model. FEMS Microbiol Lett 2005, 242:101-108.

44. Schell MA, Ulrich RL, Ribot WJ, Brueggemann EE, Hines HB, Chen D, Lipscomb L, Kim HS, Mrázek J, Nierman WC, Deshazer D: Type VI secretion is a major virulence determinant in Burkholderia mallei. Mol Microbiol 2007, 64(6):1466-1485.

45. Burtnick MN, Brett PJ, Harding SV, Ngugi SA, Ribot WJ, Chantratita N, Scorpio A, Milne TS, Dean RE, Fritz DL, Peacock SJ, Prior JL, Atkins TP DeShazer D: The cluster 1 type VI secretion system is a major virulence determinant in Burkholderia pseudomallei. Infect Immun 2011, 79(4):1512-1525

46. Stevens MP, Stevens JM, Jeng RL, Taylor LA, Wood MW, Hawes $P$, Monaghan P, Welch MD, Galyov EE: Identification of a bacterial factor required for actin-based motilityof Burkholderia pseudomallei. Mol Microbiol 2005, 56(1):40-53. 
47. Jacob-Dubuisson F, Buisine C, Mielcarek N, Clément E, Menozzi FD, Locht C: Amino-terminal maturation of the Bordetella pertussis filamentous hemagglutinin. Mol Microbiol 1996, 19(1):65-78

48. Abramson T, Kedem H, Relman DA: Proinflammatory and proapoptotic activities associated with Bordetella pertussis filamentous hemagglutinin. Infect Immun 2001, 69(4):2650-2658.

49. Dowling AJ, Wilkinson PA, Holden MT, Quail MA, Bentley SD, Reger J, Waterfield NR, Titball RW, Ffrench-Constant RH: Genome-wide analysis reveals loci encoding anti-macrophage factors in the human pathogen Burkholderia pseudomallei K96243. PLOS One 2011, 5(12):e15693.

50. Tolman JS, Valvano MA: Global changes in gene expression by the opportunistic pathogen Burkholderia cenocepacia in response to internalization by murine macrophages. BMC Genomics 2012, 13:63.

51. Hayashi F, Smith KD, Hawn TR, Yi EC, Goodlett DR, Eng JK, Akira S, Underhill DM, Aderem A: The innate immune response to bacterial flagellin is mediated by Toll-like receptor 5. Nature 2001, 410:1099-1103.

52. Feterl M, Govan BL, Ketheesan N: The effect of different Burkholderia pseudomallei isolates of varying levels of virulence on toll-like-receptor expression. Trans R Soc Trop Med Hyg 2008, 102(Suppl 1):S82-S88.

53. Chin CY, Monack DM, Nathan S: Genome wide transcriptome profiling of a murine acute melioidosis model reveals new insights into how Burkholderia pseudomallei overcomes host innate immunity. BMC Genomics 2010, 11:672.

54. Nanagara R, Vipulakorn K, Suwannaroj S, Schumacher HRJ: Atypical morphological characteristics and surface antigen expression of Burkholderia pseudomallei in naturally infected human synovial tissues. Mod Rheumatol 2000, 10:129-136.

55. Loprasert S, Whangsuk W, Sallabhan R, Mongkolsuk S: DpsA protects the human pathogen Burkholderia pseudomallei against organic hydroperoxide. Arch Microbiol 2004, 182(1):96-101.

56. Loprasert S, Sallabhan R, Whangsuk W, Mongkolsuk S: Compensatory increase in ahpC gene expression and its role in protecting Burkholderia pseudomallei against reactive nitrogen intermediates. Arch Microbiol 2003, 180(6):498-502.

57. Lee SH, Chong CE, Lim BS, Chai SJ, Sam KK, Mohamed R, Nathan S: Burkholderia pseudomallei animal and human isolates from Malaysia exhibit different phenotypic characteristics. Diagn Microbiol Infect Dis 2007, 58(3):263-270.

58. Abu Kwaik Y, Eisenstein Bl, Engleberg NC: Phenotypic modulation by Legionella pneumophila upon infection of macrophages. Infect Immun 1993, 61(4):1320-1329.

59. Bergman NH, Anderson EC, Swenson EE, Janes BK, Fisher N, Niemeyer MM, Miyoshi AD, Hanna PC: Transcriptional profiling of Bacillus anthracis during infection of host macrophages. Infect Immun 2007, 75(7):3434-3444

60. Dennis G Jr, Sherman BT, Hosack DA, Yang J, Gao W, Lane HC, Lempicki RA: DAVID: database for annotation, visualization, and integrated discovery. Genome Biol 2003, 4(5):P3.

61. Pfaffl MW, Horgan GW, Dempfle L: Relative expression software tool (RESTO) for group-wise comparison and statistical analysis of relative expression results in real-time PCR. Nucleic Acids Res 2002, 30(9):e36.

doi:10.1186/1471-2164-13-328

Cite this article as: Chieng et al:: Burkholderia pseudomallei

transcriptional adaptation in macrophages. BMC Genomics 2012 13:328.

\section{Submit your next manuscript to BioMed Central and take full advantage of:}

- Convenient online submission

- Thorough peer review

- No space constraints or color figure charges

- Immediate publication on acceptance

- Inclusion in PubMed, CAS, Scopus and Google Scholar

- Research which is freely available for redistribution 